\title{
Medida da Convergência de Prioridades em Planejamento Participativo: Indicador de Sustentabilidade em Sistemas de Interesses - ISSI
}

\author{
Luiz Renato D'Agostini ${ }^{1}$ \\ Alfredo Celso Fantini
}

Resumo: Distintos ordenamentos de prioridades às demandas compartilhadas por vários interessados geram um complexo sistema de interesses. A eloqüência em argumentar pode viciar o ordenamento coletivo das prioridades e desestimular alguns a engajaram-se no atendimento das diversas demandas. Supõe-se que a motivação do grupo em implementar ações é proporcional à compatibilidade dos ordenamentos originais ou dos re-ordenamentos a partir da reflexão individual sobre o que os outros priorizam. No método aqui proposto, a identificação do grau de conformidade entre distintos ordenamentos de prioridades é produto da matematização de relações entre os diversos ordenamentos originais, enquanto que a promoção de maior compatibilização desses ordenamentos é mediada exclusivamente pela reflexão individual. Como indicador de sustentabilidade a derivar, o grau de compatibilidade dos diversos interesses que orientam os distintos ordenamentos é tomado como uma medida das possibilidades de efetivo engajamento de todos os interessados. As relações sistematizadas resultaram num objetivo Indicador de Sustentabilidade em Sistemas de Interesses - $0 \leq \mathrm{ISSI} \leq 1$.

Palavras-chaves: indicadores de sustentabilidade; sistemas de interesses

\footnotetext{
${ }^{1}$ Professor Doutor, Universidade Federal de Santa Catarina.dagostin@mbox1.ufsc.br

${ }^{2}$ Professor Doutor, Universidade Federal de Santa Catarina.afantini@cca.ufsc.br
} 


\section{Classificação JEL: C42}

Abstract: Distinct orders of priorities for the demands shared by diverse stakeholders create a complex system of interests. The ability to argue can bias the collective ranking of priorities, discouraging people to get engaged in the fulfillment of the diverse demands. It is supposed that the motivation of a group in implementing actions is proportional to the compatibility of the original ranking or the re-ranking of priorities based on individual reflections regarding what others give priority. In the method here proposed, the identification of the degree of conformity among distinct interests is a mathematical product of the relations among the original rankings, while the promotion of a higher convergence of these rankings is mediated exclusively by individual reflection. As an indicator of sustainability to be derived, the degree of compatibility of the distinct interests that drive the distinct rankings is characterized as a measure of the possibilities of effective engagement of all interested. The systematization of relations resulted in an objective and operational Indicator of Sustainability in a System of Interests $-0 \leq$ ISSI $\leq 1$.

Keywords: indicator of sustainability; systems of interests

\section{JEL Classification: C42}

\section{1 - Introdução}

Vários parâmetros e variáveis têm sido apontados para inferir as possibilidades de sustentar processos produtivos. A esses parâmetros e variáveis tem sido atribuída a propriedade de indicador de sustentabilidade (Faema, 1997; Girardin et al. 1999; Smith et al., 2000; Bockstaller \& Girardin, 2003, p. 640). Todavia, como apontam Clayton e Radcliffe (1997, p. xiii), alguns conceitos de sustentabilidade são mesmo incompatíveis entre si. A dificuldade parece não estar em apontar indicadores, mas em delimitar conceitualmente o que se quer indicar, ou seja, dispor de um suficiente conceito de sustentabilidade - talvez por isso a fragmentação conceitual de sustentabilidade ecológica, social, econômica, política...

O tardio surgimento da palavra sustentabilidade no Século XX (Instituto 
A. Houaiss, 2001) recomenda reconhecer na expressão um significado ainda em construção. Nós propomos que, pelo menos aqui, sustentabilidade seja compreendida como uma propriedade emergente em relações presentes entre distintos interessados numa mesma questão. Mais do que perenidade de relações produtivas, sustentabilidade é aqui entendida como medida do estado da compatibilização de prioridades na sustentação do operar de um sistema de interesses com múltiplos interessados.

Não há, neste artigo, qualquer intenção em fundamentar como emergem e se processam os interesses humanos. Pressupõe-se, contudo, que um sistema de interesses resulta de múltiplas relações sociais com significados diversos e de distintas significações atribuídas a semelhantes relações. Enfim, como conceitualmente apontam Morin (1996, p.257-75), Luhmann (1997, p.38-48) e Checkland (1999, p.a10-a11), biologicamente explicam Maturana e Varella (1995, p. 83) e didaticamente exemplifica Vasconcellos (2003, p.164), a noção de sistema emerge mais como produto de distinções feitas por um observador do que a partir de estruturas ou relações cuja existência desde sempre o caracterizariam. No sistema sociedade humana, por exemplo, o ser interessado tanto pode emergir como componente distinguido no sistema de interesses como o observador que distingue o sistema.

Para o encaminhamento participativo de soluções para uma questão com outros compartilhada, é necessário valorizar todos os olhares e aspectos a partir dos quais a questão é distinguida. Garantir as condições para a manifestação de todos os interessados na questão é, assim, da maior relevância. Todavia, entre certos interessados a dificuldade maior não é apontar aspectos ou temas a partir da participação de todos em um processo sustentado na argumentação, mas evitar que a habilidade ou mesmo a simples eloqüência de alguns em argumentar determine a ordem de prioridades. Abordagens e procedimentos que procuram garantir essas condições, pela mediação do processo de seleção e de priorização de aspectos ou temas relevantes à questão, já são bem presentes na literatura como, por exemplo, em Geilfus (1997).

A partir de métodos como os discutidos ou propostos pelo autor acima é quase sempre fácil apontar temas relevantes na manifestação de um sistema de interesses. Menos fácil, porém, é promover a emergência de um genuíno ordenamento coletivo de prioridades para esses temas 
a partir de distintos interesses presentes no conjunto de interessados. Ainda menos fácil é compatibilizar e garantir efetividade aos diferentes ordenamentos originais de prioridades na obtenção de um único ordenamento. E apesar da boa intenção de quem promove um processo participativo, é questionável a condição de moderador (des)interessado, isento de preferências ou de subordinações na mediação do ordenamento de prioridades de outros. Ademais, é ainda inviável dispor-se de suficientes moderadores com eventual preparação suficiente.

A opção por um ordenamento de prioridades envolve uma decisão. Como aponta Bana e Costa (1995), nos processos de apoio à decisão são identificáveis duas fases: a estruturação e a avaliação do problema. Na estruturação, fase em que metodologias participativas como a descrita em "Soft Systems Methodology" (Checkland, 1999, Cap. 6 e 7) revelam-se especialmente eficazes, são caracterizados e organizados fatores considerados relevantes pelos interessados. Já a fase de avaliação no processo de decisão implica uma escolha. Para essa fase, métodos multicritério são relevantes à possibilidade de modelar e incorporar critérios objetivos e mesmo subjetivas preferências. Todavia, como aponta Detoni (1996), "os atores têm diferentes graus de ingerência no processo de decisão". Portanto, mesmo em métodos participativos com ou sem o recurso de métodos multicritério, a inconveniência de diferenças de poder entre interessados, entre elas a decorrente da eloqüência, é um obstáculo ao efetivo engajamento de todos os interessados em implementar o que for decidido.

Para que todos reconheçam como apropriado um ordenamento de prioridades, tão importante quanto ordenar a partir da argumentação de todos é cada um poder inferir e refletir sobre o grau de convergência entre o seu e os demais ordenamentos. É a oportunidade de cada interessado livremente refletir sobre o índice de convergência entre suas prioridades e aquelas dos demais interessados, que pode levá-lo a reordenar suas prioridades e a um efetivo engajamento nas prioridades do coletivo. O ser humano é componente de um sistema social que tanto o enriquece em sua autonomia quanto o constrange em suas liberdades e preferências. É, assim, o nível de conformidade ou valorização de distintos interesses, presentes nas relações de autonomia e restrições imanentes às relações sociais humanas, que situa o ser consciente e pode determinar o seu 
livre e efetivo engajamento em prioridades que só em parte coincidem com as suas - um pressuposto no processo participativo.

O objetivo neste artigo é derivar uma medida do grau de convergência entre ordenamentos originais ou re-ordenamentos de prioridades individuais e o ordenamento coletivo de prioridades para os mesmos temas relevantes numa situação-problema.

\section{2 - Fundamentos da proposição}

Diante de temas importantes para o interesse comum entre todos os interessados numa mesma questão, um indivíduo tanto pode apontar prioridades em uma ordem coincidente quanto muito distinta daquela de outros indivíduos. Distintas ordens de prioridades atribuídas a um conjunto de temas produzem um sistema de interesses. A emergência de satisfação entre os diversos interessados numa mesma questão requer mútuo reconhecimento de conformidade com distintos ordenamentos de prioridades. E o aumento ou mesmo a sustentação do grau de satisfação requer um mínimo de simetria entre os graus de conformidade.

Já se apontou que o objeto deste artigo não é discutir a emergência do interesse. Todavia, o que se pressupõe a seguir é importante para facilitar a exposição do que se propõe, independentemente da gênese da manifestação do interesse. Cada interessado que pensa poder justificar determinado ordenamento de prioridades mantém variados níveis de vínculos de diferentes naturezas (afetiva, familiar, profissional, institucional, cultural, ideológica, epistêmica) com os outros interessados numa mesma questão. Admita-se que, tão ou mais que o conteúdo do tema, são os vínculos entre interessados em relação a ele que determinam a semelhança de significado ou de importância atribuída. Admite-se, então, que entre $n_{i}(i=1,2 \ldots N)$ interessados ou categorias de interessados numa mesma questão e cujos vínculos relevantes a um tema enfraquecem idealmente de $\mathrm{n}_{\mathrm{i}=1}$ para $\mathrm{n}_{\mathrm{i}=\mathrm{N}}$, a conformidade com a prioridade máxima atribuída a esse tema por parte da categoria $n_{i=1}$ normalmente decresce em taxa decrescente na medida em que enfraquecem esses vínculos entre os interessados dessa categoria e os interessados das demais categorias (Figura 1a). De outro lado, a conformidade com a prioridade máxima coletiva do grupo de $\mathrm{n}_{\mathrm{i}=\mathrm{N}-1}$ interessados normalmente será decrescente 
em taxa crescente na medida em que se fortalecem aqueles vínculos com os interessados da categoria $\mathrm{n}_{\mathrm{i}=1}$ (Figura $1 \mathrm{~b}$ ).

Figura 1. Conformidade (GC) com a prioridade individual (a) e com a prioridade coletiva (b), para ni $(i=1,2 \ldots N)$ categorias de interessados, quando os vínculos relevantes entre eles enfraquecem idealmente de 1 para $\mathrm{N}$.
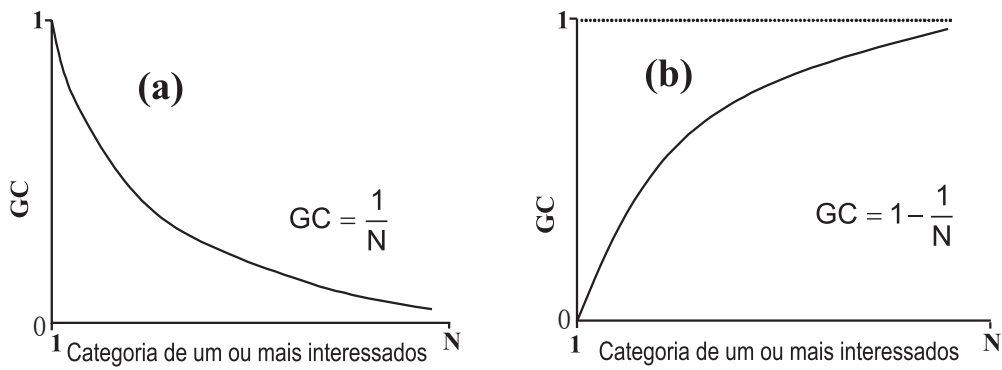

\section{3 - Sistematização das relações}

Do que se pressupôs até aqui, um Indicador de Sustentabilidade num Sistema de Interesses (ISSI) deve ser derivado do produto entre o presente estado U de satisfação de interessados e as possibilidades T de todos estarem igualmente dispostos a sustentar ou promover relações das quais emerge e pode aumentar o estado de satisfação U, ou seja,

$$
\text { ISSI }=f(U x T),
$$

uma vez que pouco significa sustentar relações que não promovam satisfação de interessados e é insuficiente sustentar relações que não promovam satisfação ao conjunto de interessados.

Como se verá mais adiante, diversos ordenamentos de prioridades de interessados de uma categoria definida a priori podem ser sintetizados em um único ordenamento e, portanto, representar uma única categoria de interesse. Não está excluída, assim, a possibilidade de que os ordenamentos de prioridades individuais de dois interessados a priori de uma mesma categoria venham divergir mais do que divergem ordenamentos de interessados de categorias tidas como distintas. Então, seja expressando o interesse de uma categoria de múltiplos interessados ou o interesse de um único interessado, um determinado ordenamento de prioridades constitui 
uma categoria de interesse. Uma vez que se admite que ordenamentos de prioridades de dois interessados de uma mesma categoria podem diferir mais entre si do que diferem ordenamentos de dois interessados de categorias a priori distintas, avaliar a satisfação coletiva U demanda avaliar a compatibilidade entre categorias de interesses que emanam de distintos interessados, mais do que avaliar a compatibilidade entre categorias de interessados. Categorias de interesses numa mesma questão se distinguem, portanto, pela diferença no ordenamento de prioridades de categorias de interessados compostas de um ou mais indivíduos.

Um conjunto $\alpha_{\mathrm{w}}(\mathrm{w}=1,2 \ldots q)$ de temas ordenados segundo os seus graus $p$ de prioridade por $\mathrm{n}_{\mathrm{i}}(\mathrm{i}=1,2 \ldots \mathrm{N})$ interessados ou categorias de interessados produzirá $\mathrm{C}_{\mathrm{j}}(\mathrm{j}=1,2 \ldots k)$ diferentes ordenamentos. Os $k$ ordenamentos correspondem ao número de categorias de interessados efetivamente distintas, ou seja, o número de categorias de interesses presentes no sistema de interesses, tal que $1 \leq k \leq \mathrm{N}$ quando $\mathrm{N} \leq q$ ! e $1 \leq k \leq q$ ! quando $\mathrm{N}>q$ ! .

Considere-se agora somente dois temas A e B de uma questão. O tema $\mathrm{A}$, prioritário para determinada categoria $\mathrm{n}_{\mathrm{i}=1}$ de interessados, tende a merecer um grau de prioridade cada vez mais baixo na medida em que esse grau é atribuído por outras categorias e cujos vínculos com a primeira e em relação a esse tema enfraquecem (Quadro 1). De outro lado, o tema $\mathrm{B}$, com grau máximo de prioridade para os integrantes da categoria $n_{i=N}$, tende a merecer graus de prioridade cada vez mais baixos na medida em que esses graus são atribuídos por categorias cujos vínculos com $\mathrm{n}_{\mathrm{i}=1}$ e em relação ao tema se fortalecem.

Quadro 1. Graus $p$ de prioridade* para os temas A e B entre $k$ categorias de interesses presentes entre $\mathrm{N}$ interessados e cujos vínculos entre si enfraquecem idealmente de $n_{1}$ para $n_{N}$.

\begin{tabular}{c|c|c|c|c|c|c|c|c}
\multicolumn{10}{c}{ Categoria de interessados } \\
\cline { 2 - 10 } Tema & $\mathrm{n}_{1}$ & $\mathrm{n}_{2}$ & $\mathrm{n}_{3}$ & $\mathrm{n}_{4}$ & $\mathrm{n}_{5}$ & $\mathrm{n}_{6}$ & & $\mathrm{n}_{\mathrm{N}}$ \\
$\mathrm{A}$ & 1 & 2 & 3 & 4 & 5 & 6 & $\ldots$ & $\mathrm{k}=\mathrm{N}$ \\
\hline $\mathrm{B}$ & $k=\mathrm{N}$ & $k-1$ & $k-2$ & $k-3$ & $k-4$ & $k-5$ & $\ldots$ & 1 \\
\hline
\end{tabular}

* O grau de prioridade decresce de 1 para $k$ 
Como se verá a seguir, um procedimento análogo àquele usado na obtenção da estatística Qui-quadrado $\left(\chi^{2}\right)$ possibilita quantificar o desaparecimento de similaridade entre interesses a partir de graus de prioridade atribuídos para um mesmo tema. O afastamento ou falta de conformidade verificada entre graus de prioridade atribuídos ao mesmo tema por duas categorias de interessados muito distintas equivale ao somatório de incrementos de afastamentos entre os graus de prioridade atribuídos àquele tema pelas diversas e subseqüentes $n_{i}(i=1,2 \ldots N)$ categorias de interessados. O incremento unitário no afastamento do grau de prioridade entre duas categorias de interessados ideal e imediatamente subseqüentes define a taxa de afastamento entre as $C_{j}$ categorias de interesses. Essa taxa de afastamento entre categorias de interesses, por sua vez, define uma função grau de conformidade reconhecida pelos integrantes das diversas categorias à prioridade máxima de determinada categoria. Então, na analogia ao $\chi^{2}$ aqui eleita, e considerando o que está pressuposto nas relações representadas no Quadro 1, ou seja, que o grau de prioridade esperado para determinado tema por parte de portadores de um interesse é o grau que eles atribuem, a função Grau de Conformidade GC em função do que percebem as diferentes categorias de interessados pode ser dada como

$$
G C_{i=1}=\frac{\left(p_{o n_{i+1}}-p_{e n_{i}}\right)^{2}}{p_{e n_{i}}} \Leftrightarrow \frac{\left(C_{j+1}-C_{j}\right)^{2}}{C_{j}}=\frac{1}{C_{j}}
$$

em que $\mathrm{GC}_{\mathrm{i}=1}$ é o grau de conformidade reconhecida por parte de cada uma das categorias à prioridade máxima de uma determinada categoria $\left(\mathrm{n}_{\mathrm{i}=1}\right)$, enquanto $\mathrm{p}_{\mathrm{o}} \mathrm{n}_{\mathrm{i}+1}$ e $\mathrm{p}_{\mathrm{e}} \mathrm{n}_{\mathrm{i}}$ são, respectivamente, grau de prioridade observado (atribuído pelos portadores do interesse de categoria $\mathrm{C}_{j+1}$ ) e grau de prioridade esperado pelos portadores do interesse de categoria $\mathrm{C}_{j}$ para o tema A. Por analogia e como está exemplificado no Quadro 1, para um tema B prioritário para interessados de uma categoria $n_{i=N}$ e em relação ao qual os mesmos mantêm os mais fracos vínculos com os interessados daquela determinada categoria $n_{i=1}$, o grau de conformidade $\mathrm{GC}_{\mathrm{i}=\mathrm{N}}$ por parte de portadores de interesses de categorias $C_{j}(j=1,2 \ldots k-1)$ então será

$$
G C_{i=N}=1-\frac{\left(p_{o n_{i-1}}-p_{e n_{i}}\right)^{2}}{p_{e n_{i}}} \Leftrightarrow 1-\frac{\left(C_{j-1}-C_{j}\right)^{2}}{C_{j}}=1-\frac{1}{C_{j}}
$$


As relações sistematizadas nas Equações (2) e (3), referentes às categorias de interesses com semelhança decrescente de $\mathrm{j}=1$ para $\mathrm{j}=k$, são análogas às ilustradas na Figura 1 e referentes a categorias de interessados com vínculos que enfraquecem idealmente de 1 para $\mathrm{N}$.

\section{4 - Obtendo o fator U: medida do estado de satisfação presente}

Como se verá mais adiante, com expressões análogas às Equações (2) e (3) pode-se obter o distanciamento de conformidade entre dois ordenamentos de graus de prioridade para qualquer número $q$ de temas. Ou seja, dado o ordenamento que representa o interesse de determinada categoria de interessados, o procedimento permite confrontá-lo com o ordenamento que representa o interesse coletivo das demais N-1 categorias de interessados (Quadro 2). Nesse interesse coletivo estarão contidos $1 \leq k \leq \mathrm{N}$-1 interesses (no caso, 1), que poderão ser desde muito semelhantes ou mesmo iguais ao interesse da categoria $\mathrm{n}_{\mathrm{i}=1}$, até muito semelhantes ou mesmo iguais ao interesse de $\mathrm{n}_{\mathrm{i}=\mathrm{N}}$ (no caso, igual ao de $\mathrm{n}_{\mathrm{i}=\mathrm{N}}$ ).

Quadro 2. Prioridades para $q(A, B \ldots E)$ temas para cinco categorias de interessados e prioridade média de cada tema para o conjunto de N-1 categorias*

\begin{tabular}{c|c|c|c|c|c|c}
\hline \multirow{2}{*}{ Tema } & \multicolumn{6}{|c}{ Categoria de interessados } \\
\cline { 2 - 7 } & $\mathrm{n}_{\mathrm{i}=1}$ & $\mathrm{n}_{\mathrm{i}=2}$ & $\mathrm{n}_{\mathrm{i}=3}$ & $\mathrm{n}_{\mathrm{i}=4}$ & $\mathrm{n}_{\mathrm{i}=5}$ & Média $\mathrm{n}_{\mathrm{i}=2 \mathrm{a} 5}$ \\
\hline $\mathrm{A}$ & 4 & 5 & 5 & 5 & 5 & 5 \\
\hline $\mathrm{B}$ & 1 & 2 & 2 & 2 & 2 & 2 \\
\hline $\mathrm{C}$ & 2 & 4 & 4 & 4 & 4 & 4 \\
\hline $\mathrm{D}$ & 3 & 3 & 3 & 3 & 3 & 3 \\
\hline $\mathrm{E}$ & 5 & 1 & 1 & 1 & 1 & 1 \\
\hline
\end{tabular}

* As prioridades das categorias 2, 3, 4 e 5 são iguais para facilitar a exposição da proposição. Em 7 será discutido como definir a prioridade média de vários interessados ou categorias de interessados.

Na sustentação de suas respectivas prioridades, tanto os interessados portadores de um determinado interesse $C_{j=1}$ quanto aqueles portadores de qualquer dos demais distintos interesses podem pensar e agir de forma mais ou então menos convergente, fazendo com que as respectivas prioridades resultem com variáveis graus de conformidade GC. A emergência de conformidade entre interesses implica a existência dos parâmetros $r$ e $s$ representados na Figura 2 . 
Figura 2. Satisfação a/(N-1) entre N categorias de interessados em função da Conformidade (GC) reconhecida por portadores de k categorias de interesses às ordens de prioridades de uma $\left(\mathrm{n}_{\mathrm{i}}={ }_{1} \Leftrightarrow \mathrm{I}\right)$ e de um grupo de categorias $\left(\mathrm{n}_{\mathrm{i}}={ }_{\mathrm{N}-1} \Leftrightarrow \mathrm{M}\right)$ de interessados.

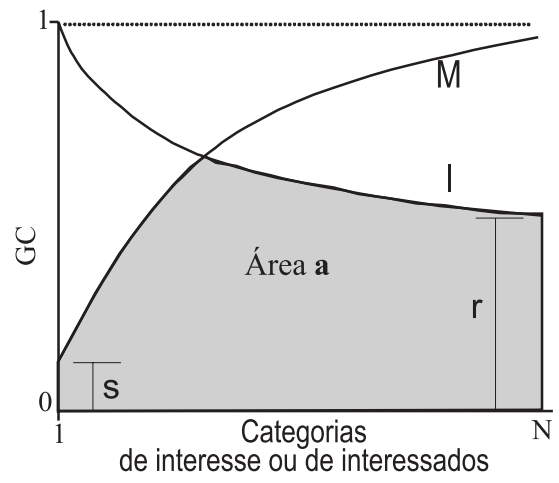

Os valores de $r$ e de $s$ são medidas do mútuo reconhecimento de pertinência entre os dois ordenamentos de prioridades, ou seja, medidas da conformidade entre o interesse de uma categoria e o interesse coletivo das demais. Tanto $r$ quanto $s$ seriam nulos quando não ocorresse qualquer reconhecimento mútuo de pertinência em apontar as mais altas e as mais baixas prioridades entre os q temas. O estado de satisfação dos interessados (U) em relação àqueles dois interesses (ordenamentos) cresce, portanto, com o crescimento dos parâmetros $r$ e $s$. U cresce, assim, proporcionalmente ao crescimento da área $a$. Essa área $a$, por sua vez, é sempre uma fração de uma área A definida pelas coordenadas $(1,0),(\mathrm{N}, 0),(1,1)$ e $(\mathrm{N}, 1)$. Para $r$ e $s$ nulos ainda ocorre um mínimo de área $a$, significando que um máximo de discordância entre dois ordenamentos de prioridades não exclui a possibilidade de alguns interesses satisfeitos. Então, sempre $\mathrm{U}>0$. De outro lado, se $r$ e $s$ são unitários significa um único interesse, um único ordenamento de prioridades. Então, U $\leq 1$. Assim,

$$
0<U \leq 1 \quad \text { ou } \quad 0<U=\frac{a}{A} \geq \frac{\frac{r+s}{2} \cdot(N-1)}{(N-1) \cdot 1}=\frac{r+s}{2} \leq 1
$$

As funções I e M na Figura 2 são, respectivamente, as funções sugeridas na Figura 1 e definidas nas Equações (2) e (3), a elas incorporados os parâmetros $r$ e $s$, ou seja, 


$$
\begin{aligned}
& I=\frac{1}{C}-\frac{r}{C}+r \quad \therefore \quad I=\frac{1}{C}-r\left(\frac{1}{C}-1\right) \\
& e \\
& M=1-\frac{1}{C}+\frac{s}{C} \quad \therefore M=1-\frac{1}{C}(1-s)
\end{aligned}
$$

\section{Derivando valores de s e de $\mathrm{r}$}

Para facilitar a exposição da proposição, na medida em que as relações tratadas neste texto resultem sistematizadas, de ora em diante os dados do Quadro 2, mais especificamente os ordenamentos de prioridades da categoria $\mathrm{n}_{\mathrm{i}=1}$ e de prioridades médias do conjunto $\mathrm{n}_{\mathrm{i}=2 \text { a } 5}$, serão, até a Equação (18), desde logo aplicados na exemplificação das relações propostas.

Como já se apontou e se procede a seguir, na analogia traçada pode-se caracterizar o afastamento de conformidade entre aqueles dois ordenamentos de prioridades para os temas A, B, C, D e E no Quadro 2. Por força da analogia ao $\chi^{2}$, à luz das prioridades dos interessados da categoria $n_{i=1}$ a ordem esperada para os graus $p$ de prioridade é, evidentemente, a sua. O mesmo é verdade para a ordem esperada na categoria de interesse do conjunto de N-1 interessados. Então, na forma genérica (e quando a partir dos dados do Quadro 2),

$$
\Delta_{k / 1}=\sum_{w=1}^{q}\left(\frac{\left(p_{k-1}-p_{1}\right)^{2}}{p_{1}}\right)_{w} \Leftrightarrow\left(\frac{(5-4)^{2}}{4}+\frac{(2-1)^{2}}{1}+\frac{(4-2)^{2}}{2}+\frac{(3-3)^{2}}{3}+\frac{(1-5)^{2}}{5}=6,4\right)
$$

em que $\Delta_{\mathrm{k} / \mathrm{I}}$ é o afastamento que o conjunto dos $k$-1 ordenamentos assume em relação ao ordenamento 1 , enquanto que $\mathrm{p}_{\mathrm{k}-1}$ e $\mathrm{p}_{1}$ são, respectivamente, os graus de prioridade $p$ atribuídos pelos interessados das N-1 categorias (grau de prioridade observado) e pela categoria $\mathrm{n}_{\mathrm{i}=1}$ (grau esperado) aos mesmos $q$ temas. Analogamente,

$$
\Delta_{1 / k}=\sum_{w=1}^{q}\left(\frac{\left(p_{1}-p_{k-1}\right)^{2}}{p_{k-1}}\right)_{w} \Leftrightarrow\left(\frac{(4-5)^{2}}{5}+\frac{(1-2)^{2}}{2}+\frac{(2-4)^{2}}{4}+\frac{(3-3)^{2}}{3}+\frac{(5-1)^{2}}{1}=17,7\right)
$$

em que $\Delta_{1 / \mathrm{k}}$ é o afastamento do ordenamento 1 em relação àquele do conjunto de k-1 para os mesmos $q$ temas, enquanto que $\mathrm{p}_{1}$ e $\mathrm{p}_{\mathrm{k}-1}$ são, respectivamente, graus $p$ observados e esperados. 
Os valores de $r$ e de $s$ também são, por definição, frações de um afastamento máximo $\Delta_{\text {máx. }}$ possível entre dois ordenamentos de prioridades (Figura 3). Um afastamento $\Delta_{\text {máx. }}$ resulta quando os ordenamentos são simetricamente opostos $(\mathrm{r}=\mathrm{s}=0)$. Procedendo da mesma forma que na obtenção de $\Delta_{\mathrm{k} / 1}$ e $\Delta_{1 / \mathrm{k}}$, e dado que para $q$ temas que recebem distintos graus $p$ de prioridade o número desses graus é sempre igual a $q$, o distanciamento máximo $\Delta_{\text {máx }}$ entre ordens de prioridades para $q$ temas (e para os cinco temas do Quadro 2) verifica-se ser:

$$
\Delta_{\text {máx. }}=\sum_{p=1}^{q} \frac{(q+1-2 p)^{2}}{p} \Leftrightarrow\left(\frac{(5-1)^{2}}{1}+\frac{(4-2)^{2}}{2}+\frac{(3-3)}{3}+\frac{(2-4)^{2}}{4}+\frac{(1-5)^{2}}{5}=22,2\right)
$$

Como $r$ e $s$ crescem na medida em que os afastamentos $\Delta_{\mathrm{k} / \mathrm{I}}$ e $\Delta_{1 / \mathrm{k}}$ decrescem em relação a um afastamento máximo $\Delta_{\text {máx. }}$ assimilado à unidade,

$$
\begin{aligned}
& \mathrm{r}=1-\frac{\Delta_{\mathrm{k} / 1}}{\Delta_{\text {máx. }}} \Leftrightarrow\left(1-\frac{6,4}{22,2}=0,71\right) \\
& \mathrm{e} \\
& \mathrm{s}=1-\frac{\Delta_{1 / \mathrm{k}}}{\Delta_{\text {máx. }}} \Leftrightarrow\left(1-\frac{17,7}{22,2}=0,20\right) .
\end{aligned}
$$

Figura 3. Valores de $\mathrm{r}$ e de $\mathrm{s}$ respectivamente tomados como a diferença entre os afastamentos $\Delta \mathrm{k} / 1$ e $\Delta 1 / \mathrm{k}$ verificados e o afastamento máximo possível $\Delta$ máx.

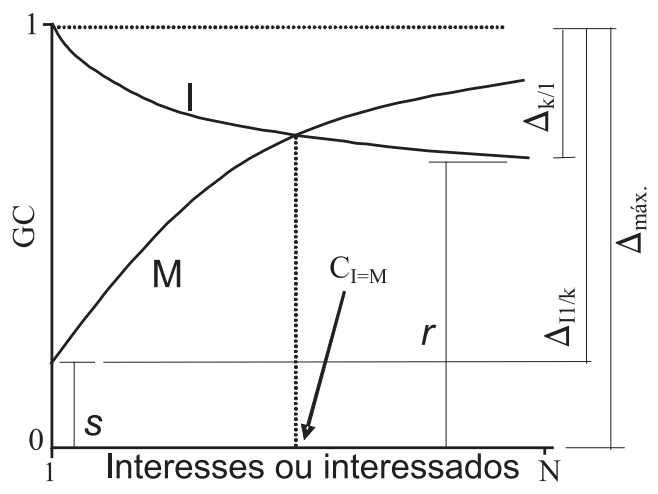




\section{Definindo a área $a$}

A área $a$ (Figura 2) é obtida da integração das funções M (Equação 6) e I (Equação 5) nos intervalos $\left[1, C_{I=M}\right]$ e $\left[C_{I=M}, N\right]$, respectivamente (vide Figura 3). Igualando as Equações (5) e (6) e expressando essa igualdade em função de $r$ e de $s$ pode-se definir $\mathrm{C}_{\mathrm{I}=\mathrm{M}}$ como

$$
\frac{1}{C}-r\left(\frac{1}{C}-1\right)=1-\frac{1}{C}(1-s) \therefore C_{l=M}=\frac{2-r-s}{1-r} \Leftrightarrow\left(\frac{2-0,71-0,20}{1-0,71}=3,76\right) .
$$

Então, (e lembrando que no Quadro $2 \mathrm{~N}=5$ )

$$
\begin{aligned}
& \mathrm{a}=\mathrm{C}-\ln \mathrm{C}+\left.\mathrm{s} \cdot \ln \mathrm{C}\right|_{1} ^{\mathrm{C}_{1=\mathrm{M}}}+\ln \mathrm{C}-\mathrm{r} \cdot \ln \mathrm{C}+\left.\mathrm{r} \cdot \mathrm{C}\right|_{\mathrm{C}_{1=\mathrm{M}}} ^{\mathrm{N}} \therefore \\
& \mathrm{a}=\mathrm{C}-\ln \mathrm{C}+0,\left.2 \cdot \ln \mathrm{C}\right|_{1} ^{3,76}+\ln \mathrm{C}-0,71 \cdot \ln \mathrm{C}+0,\left.71 \cdot \mathrm{C}\right|_{3,76} ^{5} \quad(=2,67)
\end{aligned}
$$

Quando $\mathrm{C}_{\mathrm{I}=\mathrm{M}}=2(r=s)$ existem apenas duas categorias de interesses mútua e igualmente afastadas $\left(\Delta_{\mathrm{k} / 1}=\Delta_{1 / \mathrm{k}}\right)$. Situações em que $\mathrm{C}_{\mathrm{I}=\mathrm{M}} \neq 2(r \neq S)$ denotam uma assimetria entre interesses. Enquanto as mais elevadas prioridades de uma categoria de interesse merecem graus elevados de prioridade também na outra categoria, a recíproca não acontece (como no Quadro 2). $C_{I=M}>2$ denota que o ordenamento de prioridades da categoria $n_{i=1}$ afasta-se mais do ordenamento coletivo do que esse se afasta daquele. Para $\mathrm{C}_{\mathrm{I}=\mathrm{M}}<2$ a situação é a recíproca.

Uma relação em que $r>>s$ (Figura 3) não é propícia à emergência de sustentabilidade como é aqui entendida, mesmo que por força de um interesse a relação possa ser sustentada. As relações também não promovem sustentabilidade se $r<<s$. Quando $r>>s$, por exemplo e como implicam os dados do Quadro 2 e aponta o valor de 3,76 para $\mathrm{C}_{\mathrm{I}=\mathrm{M}}$, a ordem de prioridades dos portadores do interesse $\mathrm{C}_{\mathrm{j}=1}$ é percebida com elevado grau de conformidade entre as $k$ categorias de interesses presentes, mas, por alguma razão, a primeira percebe um baixo grau de conformidade na ordem coletiva de prioridades. Note que a prioridade máxima do conjunto de $\mathrm{N}-1$ categorias de interessados no Quadro 2 é apenas a quinta prioridade da categoria $\mathrm{n}_{\mathrm{i}=1}$, enquanto que a prioridade máxima dessa é a segunda prioridade daquelas. 


\section{5 - Obtendo o fator T: um coeficiente de possibilidades de sustentação do estado U}

A área $a$ é suficiente para apontar o grau de conformidade entre ordenamentos de prioridades somente quando $r=s$, ou seja, quando ocorre um mútuo e equivalente reconhecimento de conformidade entre os dois ordenamentos considerados. A diferença entre valores de $r$ e $s$ é, por definição, um afastamento de simetria entre ordenamentos de prioridades atribuídas aos mesmos $q$ temas. Então, a diferença entre $r$ e $s$ verificada tomada em relação à diferença máxima possível entre esses dois parâmetros define um grau de assimetria.

Independente de se $r=s$ ou $r \neq s$, não se pode verificar mudança no valor de um desses parâmetros sem que o valor do outro também mude. O afastamento máximo possível entre dois ordenamentos de prioridades cresce, claro, com o crescimento do número $q$ de temas ordenados. Procedendo-se de forma análoga àquela na obtenção de $\Delta_{\text {máx. }}$ (Equação 9), verifica-se que a maior diferença possível entre $r$ e $s$ ocorre quando frente a um ordenamento $1,2,3, \ldots q$, o outro ordenamento é $q, 1,2,3 \ldots q$-1 se $q \leq 6$; ou $q, q-1,1,2,3 \ldots q-2$ se $7 \leq q \leq 11$; ou $q, q-1, q-2,1,2,3 \ldots q-3$ se $12 \leq q \leq 16$ e assim por diante. Portanto, para cinco temas como no Quadro 2,

$$
\Delta_{1 / \mathrm{kmáx}}=\frac{(1-5)^{2}}{1}+\frac{(2-1)^{2}}{2}+\frac{(3-2)^{2}}{3}+\frac{(4-3)^{2}}{4}+\frac{(5-4)^{2}}{5}=17,3
$$

e

$$
\Delta_{k / 1 \text { máx }}=\frac{(5-1)^{2}}{5}+\frac{(1-2)^{2}}{1}+\frac{(2-3)^{2}}{2}+\frac{(3-4)^{2}}{3}+\frac{(4-5)^{2}}{4}=5,3
$$

em que $\Delta_{1 / \mathrm{kmáx}}$ e $\Delta_{\mathrm{k} / \mathrm{Imax}}$ correspondem respectivamente aos afastamentos a partir dos quais os valores de $s$ e $r$ produzem a maior diferença entre eles. Substituindo $\Delta_{\mathrm{k} / 1}$ por $\Delta_{\mathrm{k} / 1 \text { máx }}$ na Equação (10) e $\Delta_{1 / \mathrm{k}}$ por $\Delta_{1 / \mathrm{kmáx}}$ na Equação (11) obtém-se valores de $r^{\prime}$ e de $s^{\prime}$ correspondentes ao máximo de assimetria entre dois ordenamentos de prioridades e nas quais $r \neq s$. Ou seja,

$$
r^{\prime}=1-\frac{\left(\Delta_{k} / 1\right)_{\text {máx. }}}{\Delta_{\text {máx. }}} \Leftrightarrow\left(1-\frac{5,3}{22,2}=0,76\right)
$$


e

$$
s^{\prime}=1-\frac{\left(\Delta_{1 / k}\right)_{\text {máx. }}}{\Delta_{\text {máx. }}} \Leftrightarrow\left(1-\frac{17,3}{22,2}=0,22\right)
$$

Como já foi apontado, a razão entre os módulos da diferença $r$-s observada e da diferença potencialmente possível $r$ '-s' com $q$ temas define um coeficiente de assimetria $\lambda$, ou seja,

$$
\frac{|r-s|}{\left|r^{\prime}-s^{\prime}\right|}=\lambda \quad \Leftrightarrow \quad\left(\frac{|0,71-0,20|}{|0,76-0,22|}=0,94\right)
$$

Isso permite definir $\mathrm{T}$ - o fator possibilidades de sustentação dos ordenamentos - como

$$
\mathrm{T}=1-\lambda \Leftrightarrow(1-0,94=0,06)
$$

ou seja, enquanto o valor de T aponta o grau de mútuo reconhecimento entre os ordenamentos de prioridades, valores de $r$-s menores ou maiores do que zero respectivamente apontam se as divergências existentes decorrem mais pela dificuldade do conjunto das N-1 categorias concordarem com as prioridades máximas da categoria $\mathrm{n}_{\mathrm{i}=1}(r<s)$, ou se pela dificuldade dessa categoria concordar com as prioridades máximas do coletivo $(r>s)$.

\section{6 - O produto UxT: o Indicador de Sustentabilidade em Sistemas de Interesses - ISSI}

Do que foi apontado na Equação (1) e até aqui sistematizado, o Indicador de Sustentabilidade em Sistemas de Interesses (ISSI) resulta dado como

$$
\text { ISSI }=\frac{a}{N-1}(1-\lambda) \quad \Leftrightarrow \quad\left(\frac{2,67}{5-1}(1-0,94)=0,03\right)
$$

em que $a /(\mathrm{N}-1)$ é medida de satisfação de interesses presentes entre $\mathrm{N}$ interessados ou categorias de interessados e 1- $\lambda$ é uma medida das possibilidades de sustentar o presente estado de satisfação. Mantidas as ordens de prioridades apresentadas no Quadro 2, por exemplo, apesar do elevado valor de $U=0,67(2,67 / 4)$, as possibilidades de emergir sus- 
tentabilidade são muito baixas (3\%) em função de T muito baixo $(0,06)$, dado que os portadores do interesse $\mathrm{C}_{\mathrm{j}=1}$ atribuem baixa conformidade às prioridades do conjunto dos demais N-1 interessados.

\section{7 - Ordenamento de prioridades de temas em uma categoria com vários interessados}

Dado o número $q$ de temas, também ficam definidos o afastamento máximo $\Delta_{\text {máx. }}$ possível entre ordenamentos (Equação 9) e a soma de graus de prioridade de temas ordenados. Não há, portanto, repetição de grau de prioridade entre dois temas para uma mesma categoria quando essa está representada por um único indivíduo interessado. Somente quando uma categoria de interessados é composta de vários indivíduos é que pode resultar um ordenamento de prioridades no qual dois ou mais temas têm o mesmo grau médio de prioridade. Todavia, na forma apresentada no Quadro 3 e como se considera a seguir, um mesmo grau de prioridade atribuído a dois temas por dois interessados não suprime a necessidade da constância do valor da soma de graus de prioridade, quanto não anula o caráter referencial do termo $\Delta_{\text {máx. }}$ como máximo afastamento possível entre ordenamentos de $q$ temas (Equação (9).

Pressupõe-se que quando um indivíduo ordena um conjunto de temas segundo suas prioridades ele é mais rigoroso na distinção entre temas de elevada prioridade, ou seja, há mais reflexão na distinção entre a primeira e a segunda prioridade do que na distinção entre a oitava e a nona. A média geométrica dos diversos graus de prioridade para um tema contempla esse pressuposto na obtenção do ordenamento médio de uma categoria de múltiplos interessados. Ocorre que na média geométrica o valor mais baixo (maior prioridade) tem maior peso em determinar essa média. A média geométrica também diminui a ocorrência de valores médios coincidentes (Quadro 3). Quando dois ou mais temas resultam com o mesmo grau de prioridade médio, tanto significa que nenhum deles caracteriza uma prioridade em relação ao(s) outro(s), quanto nenhum deles pode ser caracterizado como menos prioritário. Como a soma de graus de prioridades é constante para determinado número $q$ de temas, o grau que se deve atribuir a cada um dos diferentes temas empatados em prioridade é um grau que, multiplicado pelo número de temas com 
mesma prioridade, corresponda ao somatório de graus correspondentes em um ordenamento em que não ocorre valores médios iguais. No Quadro 3 não há grau de prioridade 2 e é igual a 1,5 para os temas B e E porque duas vezes 1,5 é igual a $1+2$.

Quadro 3. Ordem de graus de prioridade de temas para uma categoria de cinco indivíduos

\begin{tabular}{c|c|c|c|c|c|c|c|c}
\hline \multirow{2}{*}{ Tema } & \multicolumn{9}{|c|}{ Interessado } & \multirow{2}{*}{ Ordem } \\
\cline { 2 - 9 } & I & II & III & IV & V & Aritmética & Geométrica & \\
\hline A & 8 & 1 & 6 & 6 & 7 & 5,6 & 4,58 & 5 \\
\hline B & 2 & 3 & 1 & 3 & 1 & 2,0 & 1,78 & 1,5 \\
\hline C & 6 & 8 & 8 & 5 & 6 & 6,6 & 6,49 & 7 \\
\hline D & 7 & 7 & 7 & 7 & 5 & 6,6 & 6,54 & 8 \\
\hline E & 1 & 2 & 3 & 1 & 3 & 2,0 & 1,78 & 1,5 \\
\hline F & 5 & 6 & 2 & 8 & 8 & 5,8 & 5,21 & 6 \\
\hline G & 4 & 4 & 4 & 2 & 4 & 3,6 & 3,48 & 3 \\
\hline H & 3 & 5 & 5 & 4 & 2 & 3,8 & 3,59 & 4 \\
Soma & 36 & 36 & 36 & 36 & 36 & & & 36 \\
\hline
\end{tabular}

\section{8 - Resultado da sistematização}

Para interpretar o produto das relações sistematizadas neste artigo e já aplicadas em situações-problema reais, entre outras como por Anton (2004) entre interessados em organizar uma associação de produtores de leite, 6 temas (A, B... F) relevantes à uma problemática são ordenados segundo sua prioridade à luz do olhar de cada um dos sete interessados (I, II... VII) (Quadro 4). Somente o próprio interessado e o moderador do processo participativo podem associar nome e respectivo número do interessado. O grau de convergência dos interesses que orientam o ordenamento de cada interessado com o ordenamento do conjunto dos demais seis interessados é o valor de ISSI correspondente.

O resultado mais evidente (Quadro 4a) é que o ordenamento do interessado III (ISSI $=0,89$ ) revela interesses que melhor se aproximam dos demais. O resultado talvez mais importante, por sua vez, está na possibilidade de cada interessado perceber-se situado no sistema de interesses a partir de suas prioridades. A partir do valor do ISSI correspondente, o 
interessado verifica em quanto suas prioridades convergem com aquelas do conjunto dos demais interessados. A partir da notação k/1 ou 1/k na coluna à direita no Quadro 4, todos e cada um podem verificar se as divergências existentes decorrem mais em face dos interesses presentes entre os demais N-1 interessados não contemplarem suficientemente as prioridades máximas do indivíduo, ou se pelo indivíduo não concordar com as prioridades máximas do interesse coletivo.

O interessado II então refletiu sobre seu baixo grau de conformidade com o restante dos interessados (ISSI $=0,33$ ), quanto o grupo refletiu sobre a prioridade máxima $(\mathrm{F})$ do interessado VI $(\mathrm{ISSI}=0,18)$. É importante notar que quando um interessado reordena suas prioridades (sombreadas no Quadro 4b) pode mudar o índice de convergência também de outros interessados em relação aos demais. No exemplo está sugerido que o interessado II de fato reviu sua ordem de prioridades; sem alterar, entretanto, suas prioridades máximas (temas A, C e D). Em consideração à prioridade máxima da categoria VI (tema F), mais dois interessados (I e VII) reordenaram suas prioridades, reconhecendo que o tema F, prioritário para VI, não pode ser de tão baixa prioridade para o grupo. Como resultado, agora é no ordenamento do interessado $V$ que emerge a propriedade de melhor representar o interesse do grupo (ISSI $=0,93$ ). Essa propriedade, emergente, de fato nunca é do ente interessado, da parte, mas do entre, da relação entre entes, entre partes. Por isso consideramos sustentabilidade uma propriedade emergente entre interessados e apresentamos o ISSI como um indicador de sustentabilidade.

O sistema de relações aqui proposto deve ser percebido como complementar a outros métodos de apoio à decisão. A incorporação operacional desse sistema ao operar de outras estruturas metodológicas de apoio à decisão constitui-se em interessante campo de investigação. 
Quadro 4. Ordem de prioridade dos temas A, B... F para os interessados I, II... VII; índice de convergência entre prioridades individuais e do coletivo (ISSI); indivíduo mais distanciado do coletivo $(1 / \mathrm{k})$ do que o coletivo em relação ao indivíduo (k/1) ou igualmente distanciados (-)*

a - ordenamentos originais

\begin{tabular}{c|c|c|c|c|c|c|c}
\hline \multirow{2}{*}{ Tema } & \multicolumn{7}{|c}{ Categoria de interessados } \\
\cline { 2 - 8 } & I & II & III & IV & V & VI & VII \\
\hline A & 3 & 1 & 4 & 6 & 5 & 5 & 2 \\
B & 2 & 6 & 3 & 2 & 1 & 2 & 1 \\
\hline C & 4 & 2 & 1 & 1 & 3 & 4 & 3 \\
\hline D & 1 & 3 & 2 & 3 & 2 & 3 & 5 \\
\hline E & 5 & 4 & 6 & 5 & 6 & 6 & 4 \\
\hline F & 6 & 5 & 5 & 4 & 4 & 1 & 6 \\
\hline
\end{tabular}

\begin{tabular}{c|c|c}
\hline entre & ISSI & \\
\hline I x coletivo & 0,86 & $\mathrm{k} / 1$ \\
\hline II x coletivo & 0,33 & $1 / \mathrm{k}$ \\
\hline III x coletivo & 0,89 & - \\
\hline IV x coletivo & 0,81 & $\mathrm{k} / 1$ \\
\hline V x coletivo & 0,83 & $1 / \mathrm{k}$ \\
\hline VI x coletivo & 0,18 & $\mathrm{k} / 1$ \\
\hline VII x coletivo & 0,73 & $1 / \mathrm{k}$ \\
\hline
\end{tabular}

b - reordenamentos a partir da reflexão sobre os ordenamentos originais

\begin{tabular}{c|c|c|c|c|c|c|c}
\hline \multirow{2}{*}{ Tema } & \multicolumn{7}{|c}{ Categoria de interessados } \\
\cline { 2 - 8 } & I & II & III & IV & V & VI & VII \\
\hline A & 3 & 1 & 4 & 6 & 5 & 5 & 2 \\
B & 2 & 4 & 3 & 2 & 1 & 2 & 1 \\
\hline C & 4 & 2 & 1 & 1 & 3 & 4 & 3 \\
\hline D & 1 & 3 & 2 & 3 & 2 & 3 & 5 \\
\hline E & 6 & 6 & 6 & 5 & 6 & 6 & 6 \\
\hline F & 5 & 5 & 5 & 4 & 4 & 1 & 4 \\
\hline
\end{tabular}

\begin{tabular}{c|c|c}
\hline Confronto & ISSI & \\
\hline I x coletivo & 0,83 & $\mathrm{k} / 1$ \\
\hline II x coletivo & 0,45 & $\mathrm{k} / 1$ \\
\hline III x coletivo & 0,89 & - \\
\hline IV x coletivo & 0,81 & $\mathrm{k} / 1$ \\
\hline V x coletivo & 0,93 & $1 / \mathrm{k}$ \\
\hline VI x coletivo & 0,29 & $\mathrm{k} / 1$ \\
\hline VII x coletivo & 0,91 & $1 / \mathrm{k}$ \\
\hline
\end{tabular}

* No aplicativo computacional desenvolvido para a aplicação do método, as notações 1/k, k/1 e o símbolo "-" são substituídos por cores distintas, facilitando a interpretação por parte dos interessados.

\section{9 - Referências Bibliográficas}

ANTON, S. L. Medida de convergência entre interesses: indicador de sustentabilidade em planejamento participativo. Florianópolis, 2004. 73 f. Dissertação (Mestrado em Agroecossistemas) - Centro de Ciências Agrárias, Universidade Federal de Santa Catarina.

BANA E COSTA, C. A. O que entender por Tomada de Decisão Multicritério ou Multiobjetivo. Florianópolis: ENE - Escola de Novos Empreendedores da UFSC, 1995.

BOCKSTALLER, C. e GIRARDIN, P. How to validate environmental in- 
dicators. Agricultural Systems v.76, p.639-53, 2003.

CHECKLAND, P. Systems thinking, systems practice. Chichester: Wiley, 1999. 330p.

CLAYTON, A. M. H. e RADCLIFFE, N. J. Sustainability: a systems approach. London: Earthscan, 1997. 258p.

DETONI, M. M. M. L. Aplicação de metodologia multicritério de apoio à decisão na definição de características de projetos de construção. Florianópolis, 1996. 184 f. Dissertação (Mestrado em Engenharia de Produção) - Departamento de Engenharia de Produção e Sistemas, Universidade Federal de Santa Catarina.

FAEMA - Fundação Municipal do Meio Ambiente. Programa de indicadores ambientais para Blumenau. Blumenau: Furb, 1997. 17p.

GEIFULS, F. 80 herramientas para el desarollo participativo: diagnóstico, planificación, monitoreo, evaluación. San Salvador: ProchalateIICA, 1997. 208p.

GIRARDIN, P.; BOCKSTALLER, C., van der WERF, H.M.G. Indicators: tools to evaluate the environmental impacts of farming systems. Journal of Sustainable Agriculture 13, 1999. p.5-21.

INSTITUTO ANTÔNIO HOUAISS. Dicionário Houaiss da língua portuguesa. Rio de Janeiro: Objetiva, 2001.

LUHMANN, N. A nova teoria dos sistemas. Porto Alegre: Editora da UFRGS, 1997. 114p.

MATURANA, H. R. e VARELLA, F. A árvore do conhecimento. Campinas: PSY, 1995. 281p.

MORIN, E. Ciência com consciência. Rio de Janeiro: Bertrand Brasil, 1996. 336p.

SMITH, O.H.; PETERSEN, G.W. e NEEDELMAN, B.A. Environmental indicators of agroecosystems. Advances in Agronomy 69, 2000. p.75-97.

VASCONCELLOS, M.J.E. Pensando o pensamento sistêmico novo paradigmático e suas implicações. Eisforia 1(2), 2003. p. 159-70.

Recebido em março de 2005 e revisto em julho de 2005 